\title{
Supersymmetric spin glass
}

\author{
S. Gukov ${ }^{\mathrm{a})}$ \\ L. D. Landau Institute for Theoretical Physics, 117334 Moscow, Russia; Institute of \\ Theoretical and Experimental Physics; 117259, Moscow, Russia
}

(Submitted 27 February 1997; resubmitted 4 April 1997)

Pis'ma Zh. Éksp. Teor. Fiz. 65, No. 8, 657-662 (25 April 1997)

The manifestly supersymmetric four-dimensional Wess-Zumino model with quenched disorder is considered at the one-loop level. The infrared fixed points of a beta function form the moduli space $\mathscr{C}=R P^{2}$, where two types of phases are found: with and without replica symmetry. While the former phase possesses only a trivial fixed point, this point become unstable in the latter phase, which may be interpreted as a spin glass phase. (C) 1997 American Institute of Physics.

[S0021-3640(97)01808-2]

PACS numbers: $12.60 . \mathrm{Jv}, 75.10 . \mathrm{Nr}$

\section{INTRODUCTION}

There are a great many field-theoretical models describing a system in quenched random fields or with random coupling constants (Refs. 1-3, etc.). In solid state physics such models naturally arise from the corresponding pure systems whenever impurities are introduced. It is interesting to extend randomness to other well-studied field theories, just as, for example, disorder was implemented into minimal conformal models in Ref. 3. It was shown in Ref. 4 and subsequent papers that stochastic equations, like field theories in the presence of random external sources, often prove to possess some hidden supersymmetry. Kurchan ${ }^{5}$ endorsed this result for spin glass dynamics. Because supersymmetry can handle perturbative corrections, such random theories are especially interesting. Such an approach will be taken in this paper.

On the other hand in field theories with manifest space-time supersymmetry the superpotential is a holomorphic function not only of the fields but also of the coupling constants. ${ }^{6}$ Therefore the couplings and fields enter the potential on an equal footing, so that it seems very natural to introduce a random (Gaussian) distribution of some couplings in the Lagrangian. But the power of supersymmetry is so strong that the superpotential gets no quantum corrections, ${ }^{6,7}$ i.e., provided that the coupling has no dynamical D-terms, integrating over it solves the problem.

In Sec. 2 we formulate a four-dimensional supersymmetric Wess-Zumino theory in a random field. In Sec. 3 the infrared fixed points of one-loop $\beta$-functions are found in the context of the replica method. Analysis of these fixed points suggests the existence of two phases on the moduli space $\mathscr{C}=R P^{2}$. Numerical evaluation of the most general expressions is eventuated in the phase diagram, which is illustrated by two simple examples in Sec. 4. Section 5 is devoted to discussions and conclusions. 


\section{WESS-ZUMINO MODEL PERTURBED BY RANDOMNESS}

It follows from the above arguments it follows that the SUSY analog of a theory with disorder must contain dynamical terms for the random field. In the present paper we consider a four-dimensional Wess-Zumino model that is the supersymmetric counterpart of the $\varphi^{4}$-model (the two theories are defined in the same critical dimension, and the scalar potential after integrating the auxiliary field in the former model is actually $\varphi^{4}$ ). Since, according to Ref. 7, Wess-Zumino theory is defined only as a low-energy field theory, we will study the Wilsonian effective action obtained by integrating over fast modes with momenta $\Lambda^{\prime}<P<\Lambda$. We thereby define a chiral superfield $\Phi=\varphi+\theta \psi+\theta^{2} F$ and a random superfield $H$. In this notation the original action is ${ }^{\mathrm{b})}$

$$
\begin{aligned}
S= & \int d^{4} x d^{2} \theta d^{2} \bar{\theta}\left(g \Phi^{+} \Phi-\Phi^{+} H-H^{+} \Phi+\frac{1}{u} H^{+} H\right)+\frac{1}{3 !} \int d^{4} x d^{2} \theta\left(\lambda_{1}^{\prime} \Phi H^{2}\right. \\
& \left.+\lambda_{2}^{\prime} \Phi^{2} H+\lambda_{3}^{\prime} \Phi^{3}+\lambda_{4}^{\prime} H^{3}\right)+ \text { h.c. }
\end{aligned}
$$

This action admits the following treatment. It may be obtained (for a given set of parameters) from the usual Wess-Zumino action by the replacement $\Phi \rightarrow \Phi+H$, as one usually does in a summation over local extrema. ${ }^{2}$

One of the most powerful methods of dealing with random fields is the replica trick, ${ }^{1}$ which we will use here to solve this "toy" model. It reduces to introducing $n$ copies (replicas) of our system, integrating out the $H$ field, and then solving $n$-replica problem and taking $n=0$ at the end of the calculations. After replication the action (1) takes the form

$$
\begin{aligned}
S= & \int d^{4} x d^{2} \theta d^{2} \bar{\theta}\left[\sum_{a=1}^{n}\left(g \Phi_{a}^{+} \Phi_{a}-\Phi_{a}^{+} H-H^{+} \Phi_{a}\right)+\frac{1}{u} H^{+} H\right] \\
& +\frac{1}{3 !} \int d^{4} x d^{2} \theta\left[\sum_{a=1}^{n}\left(\lambda_{1}^{\prime} \Phi_{a} H^{2}+\lambda_{2}^{\prime} \Phi_{a}^{2} H+\lambda_{3}^{\prime} \Phi_{a}^{3}\right)+\lambda_{4}^{\prime} H^{3}\right]+\text { h.c. }
\end{aligned}
$$

As will be shown later, the model depends only on the relative values of the lambdas, so that one can put them small enough to determine the $H$ field from the saddle-point equation on the D-term only:

$$
H=u \sum_{a=1}^{n} \Phi_{a} \text { and } H^{+}=u \sum_{a=1}^{n} \Phi_{a}^{+} .
$$

Substituting it back into (2) yields:

$$
\begin{aligned}
S= & \sum_{a, b=1}^{n} \int d^{4} x d^{2} \theta d^{2} \bar{\theta} g_{a b} \Phi_{a}^{+} \Phi_{b}+\frac{1}{3 !} \int d^{4} x d^{2} \theta\left(\sum_{a, b, c=1}^{n} \lambda_{1} \Phi_{a} \Phi_{b} \Phi_{c}\right. \\
& \left.+\sum_{a, b=1}^{n} \lambda_{2} \Phi_{a}^{2} \Phi_{b}+\sum_{a=1}^{n} \lambda_{3} \Phi_{a}^{3}\right)+ \text { h.c. }
\end{aligned}
$$


where $g_{a a}=g+3 u, g_{a \neq b}=3 u$, and the three types of vertices $\lambda_{1}=\lambda_{1}^{\prime} u^{2}+\lambda_{4}^{\prime} u^{3}$, $\lambda_{2}=\lambda_{2}^{\prime} u$, and $\lambda_{3}=\lambda_{3}^{\prime}$ mix replica indices in different ways. It is the action (4) that we are going to study.

\section{FIXED POINTS OF $\beta$ FUNCTIONS}

The renormalization group (RG) equations for $g_{a b}$ easily follow from the one-loop diagram for the pure Wess-Zumino theory: ${ }^{8}$

$$
\begin{aligned}
\frac{d g_{a b}}{d \ln \Lambda}= & \frac{1}{288 \pi^{2}}\left\{9 \lambda_{3}^{2} g_{a b}^{2}+2 \lambda_{2}^{2} \sum_{c, d=1}^{n}\left[\left(g_{a c}+g_{b c}\right) g_{c d}+g_{a c} g_{b d}\right]+3 \lambda_{2} \lambda_{3}\left[\sum _ { c = 1 } ^ { n } \left(g_{a c}^{2}\right.\right.\right. \\
& \left.\left.\left.+g_{b c}^{2}\right)+2 g_{a b} \sum_{c=1}^{n}\left(g_{a c}+g_{b c}\right)\right]+9 \lambda_{1} \lambda_{3} \sum_{c, d=1}^{n}\left(g_{a c} g_{a d}+g_{b c} g_{b d}\right)\right\}
\end{aligned}
$$

Taking into account the possible replica symmetry breaking, we take the Parisi ansatz for $g_{a b}:{ }^{1}$ the off-diagonal part of $g_{a b}$ is parametrized by an internal function $g(x)$ defined on a unit interval $x \in[0,1]$, and the diagonal part is $g_{a a}=\widetilde{g}$. The replica-symmetric case is obtained by putting $g(x)=g=$ const. The algebra of Parisi matrices $\mathbf{a}=(\widetilde{a}, a(x))$ is defined by the multiplication rule: ${ }^{1}$

$$
\begin{aligned}
\mathbf{c}=\mathbf{a b}: \quad \tilde{c}=\tilde{a} \widetilde{b}-\int_{0}^{1} d x a(x) b(x), \\
c(x)=b(x)\left[\tilde{a}-\int_{0}^{1} d x a(y)\right]+a(x)\left[\tilde{b}-\int_{0}^{1} d x b(y)\right] \\
-\int_{0}^{x} d y(a(x)-a(y))(b(x)-b(y)) .
\end{aligned}
$$

By means of this rule we get sums over replica indices that appear in (5) in the $n \rightarrow 0$ limit:

$$
\sum_{b=1}^{n} g_{a c} \rightarrow \tilde{g}-\bar{g} \sum_{c, d=1}^{n} g_{a c} g_{c d} \rightarrow(\tilde{g}-\bar{g})^{2} \sum_{b=1}^{n} g_{a c}^{2} \rightarrow \widetilde{g}^{2}-\bar{g}^{2}
$$

where

$$
\bar{g}=\int_{0}^{1} d x g(x) \text { and } \bar{g}^{2}=\int_{0}^{1} d x g^{2}(x) .
$$

As usual in spin glass theory, one deals with the problem of finding the infrared (IR) fixed points ${ }^{\mathrm{c})}$ of Eq. (5), which determine the dynamics of the system:

$$
\begin{aligned}
& \frac{3}{2} \lambda_{3}^{2} \widetilde{g}^{2}+\left(\lambda_{2}^{2}+3 \lambda_{1} \lambda_{3}\right)(\widetilde{g}-\bar{g})^{2}+\lambda_{2} \lambda_{3}\left[2 \widetilde{g}(\tilde{g}-\bar{g})+\widetilde{g}^{2}-\bar{g}^{2}\right]=0 \\
& \frac{3}{2} \lambda_{3}^{2} g^{2}(x)+\left(\lambda_{2}^{2}+3 \lambda_{1} \lambda_{3}\right)(\widetilde{g}-\bar{g})^{2}+\lambda_{2} \lambda_{3}\left[2 g(x)(\widetilde{g}-\bar{g})+\widetilde{g}^{2}-\bar{g}^{2}\right]=0 .
\end{aligned}
$$




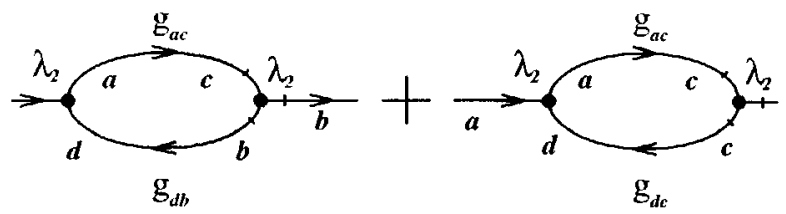

FIG. 1. Surviving (in the $n \rightarrow 0$ limit) $\lambda_{2}^{2}$-contributions.

For example, the $\lambda_{2}^{2}$ term is produced by the two nonvanishing (with number of replicas) diagrams shown in Fig. 1.

These equations have two remarkable properties: they are homogeneous in $\lambda$ and $g$, i.e., they depend only on their squares. This type of dependence on $\lambda$ tells us that the zeroes of the beta functions (9)-(10) do not depend on the values of the couplings themselves, but only on their mutual ratios, so that the moduli space of the theory is $R P^{2}$ instead of $R^{3}=\left\{\lambda_{1}, \lambda_{2}, \lambda_{3}\right\}$. Therefore, without loss of generality, we may put the couplings very small, while keeping their ratios fixed. In this limit the results that we are going to obtain are exact. Moreover, in what follows we will assume $\lambda_{3} \neq 0$, so that we can choose it to be $\lambda_{3}=1$ and denote $\lambda_{2}=\lambda$ and $\lambda_{1}=\mu$ (affined map). ${ }^{\text {d) }}$ The special case $\lambda_{3}=0$ will be studied in the first example of Sec. 4 .

Quadratic dependence on $g$ in (10) means that for each set of general characteristics, such as $\bar{g}, \bar{g}^{2}$ and $\widetilde{g}$, there are only two possible values $g_{1,2}$ (if any) which the function $g(x)$ can take on at a IR-fixed point. Moreover, the same must be true for $\widetilde{g}$, because formally it also satisfies a similar equation (9). We are free to chose $\widetilde{g}=g_{1}$, for instance. Let us denote the measure of points on a unit interval of $x$ where $g(x)=g_{1}$ as $1-x_{0}$ and the measure of points where $g(x)=g_{2}$ as $x_{0}$. For example, it may be a stepwise distribution:

$$
g(x)=\left\{\begin{array}{cc}
g_{1}, & x_{0}<x<1 \\
g_{2}, & 0<x<x_{0}
\end{array}\right.
$$

Thus we have two equations (9), (10) in three unknowns: $g_{1,2}$ and $x_{0}$, with $\bar{g}$ and $\vec{g}^{2}$ depending on them. If $g_{1}$ and $g_{2}$ are not simultaneously equal to zero ${ }^{\mathrm{e})}$ then we actually have only two unknowns: $x_{0}$ and the ratio $p=g_{2} / g_{1}$. In this notation Eqs. (9) and (10) may be rewritten as

$$
\left\{\begin{array}{c}
1+\left(\frac{2}{3} \lambda^{2}+2 \mu\right) x_{0}^{2}(1-p)^{2}+\frac{2}{3} x_{0} \lambda\left[2(1-p)+\left(1-p^{2}\right)\right]=0, \\
p^{2}+\left(\frac{2}{3} \lambda^{2}+2 \mu\right) x_{0}^{2}(1-p)^{2}+\frac{2}{3} x_{0} \lambda\left[2 p(1-p)+\left(1-p^{2}\right)\right]=0,
\end{array}\right.
$$

which determine both $p$ and $x_{0}$ and, consequently, the phase of the system.

Curiously enough, for a given solution $p$ and $x_{0}$ we get a whole set of RG-fixed points $\{\widetilde{g}, g(x)\}$, differing by an arbitrary factor. Of course, this degeneracy will be lifted by higher loop corrections, so that particular value of the fixed point will be determined 


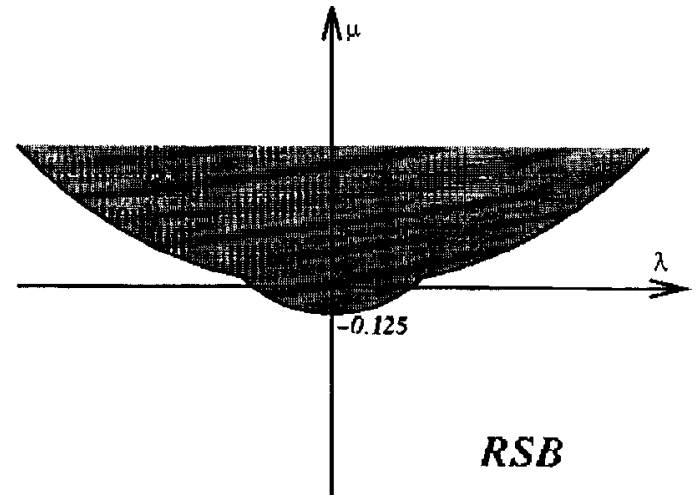

FIG. 2. The phase diagram (not drawn to scale).

by the full perturbative expansion. In the one-loop approximation, the explicit data for $(\widetilde{g}, g(x))$ at a fixed point may be determined by the initial conditions $g$ and $u$.

If for some set of couplings there is no solution to (12) except the trivial one $\widetilde{g}=g(x)=0$, we will refer to this point on the phase space $\{\lambda, \mu\} \in \mathscr{L} b=R P^{2}$ as a replica-symmetric point and will denote the corresponding phase as "RS." Otherwise, replica symmetry is broken with $x_{0}$ being the solution of (12), and the corresponding phase "RSB" looks like a spin glass system.

Since (12) must be solved by the same $p$, by equating the solutions to each equation we get a relation between $x_{0}$ and $\{\lambda, \mu\} \in \mathscr{L}$. Instead of writing the resulting complicated formula (partly because it can not be solved for $x_{0}$ ), we display it for $x_{0}=1$ :

$$
\frac{\lambda^{2}+3 \mu+\lambda \pm \sqrt{\frac{5}{2} \lambda^{2}-\frac{9}{2} \mu+\frac{3}{2} \lambda}}{\lambda^{2}+3 \mu-\lambda}=\frac{\lambda^{2}+3 \mu-\lambda \pm \sqrt{\frac{5}{2} \lambda^{2}-\frac{9}{2} \mu-\frac{3}{2} \lambda}}{\frac{3}{2}+\lambda^{2}+3 \mu-3 \lambda},
$$

where the signs in the two sides are taken independently. Replacing $\lambda \rightarrow x_{0} \lambda$ and $\mu \rightarrow x_{0}^{2} \mu$, we get Eq. (13) for arbitrary $x_{0}$. This expression describes (part of) a curve in $\mathscr{L} 6$ that separates the RS and RSB phases as shown in Fig. 2. The shaded region indicates a replica-symmetric phase and the unshaded region corresponds to replica symmetry breaking, where there is a non-trivial solution to (13), and the trivial point $\widetilde{g}=g(x)=0$ becomes unstable, as will be discussed at length in the second example of the next Section.

\section{TWO SIMPLE EXAMPLES}

a) $\lambda_{3}=0$. In this case the beta functions (5) become

$$
\frac{d \widetilde{g}}{d \ln \Lambda}=\frac{1}{48 \pi^{2}} \lambda_{2}^{2}(\widetilde{g}-\bar{g})^{2}, \quad \frac{d g(x)}{d \ln \Lambda}=\frac{1}{48 \pi^{2}} \lambda_{2}^{2}(\widetilde{g}-\bar{g})^{2} .
$$


These equations may be easily integrated, with the result:

$$
\widetilde{g}_{\Lambda}=\widetilde{g}_{0, \Lambda^{\prime}}+\frac{A}{48 \pi^{2}} \lambda_{2}^{2} \ln \frac{\Lambda}{\Lambda^{\prime}}, \quad g_{\Lambda}(x)=g_{0, \Lambda^{\prime}}(x)+\frac{A}{48 \pi^{2}} \lambda_{2}^{2} \ln \frac{\Lambda}{\Lambda^{\prime}},
$$

where the constant $A=(\widetilde{g}-\bar{g})^{2}$ is determined by the initial conditions and remains unchanged during renormalization group flow. Since for any $\lambda_{2}$ the only fixed point is $\widetilde{g}=g(x)=0$, this phase is always replica-symmetric and is not as interesting as the others.

b) $\lambda_{2}=0 \leftrightarrow \lambda=0$. Equations (9) and (10) take the form:

$$
\left\{\begin{array}{c}
\widetilde{g}^{2}+2 \mu(\widetilde{g}-\bar{g})^{2}=0 \\
g^{2}(x)+2 \mu(\widetilde{g}-\bar{g})^{2}=0
\end{array}\right.
$$

for which $g_{1,2}= \pm g$ for some $g \neq 0$ in the SG phase. In parametrization (11)

$$
\bar{g}=\left(g_{1}-g_{2}\right) x_{0}=2 g x_{0} \quad \text { and } \bar{g}^{2}=\left(g_{1}^{2}-g_{2}^{2}\right) x_{0}=2 g^{2} x_{0} \text {. }
$$

Substituting it into (14) yields a nontrivial solution:

$$
-8 \mu x_{0}^{2}=1 \quad \text { or } \quad x_{0}=\frac{1}{\sqrt{-8 \mu}}
$$

which exists only for $\mu<-1 / 8$. It is the range of $\mu$ where the RSB phase can be found. Let us emphasize that it is precisely at these points in $\mathscr{C}$ that the trivial fixed point $\widetilde{g}=g(x)=0$ becomes unstable, for example, against perturbations in $\widetilde{g}$. To see this, consider $\widetilde{g}=\epsilon$ :

$$
\frac{d \epsilon}{d \ln \Lambda}=\alpha \epsilon^{2}
$$

where $\alpha<0$ if (16) is true (i.e., an arbitrarily small $\epsilon$ increases in value during the flow to low energies). This simple case illustrates the behavior of the general system (12). On the phase diagram it corresponds to the $\mu$ axis, where both the RS and RSB phases exist.

\section{SUMMARY}

Starting from the (space-time) supersymmetric Wess-Zumino model in a random and quenched background (1), we have found that the renormalization group equations (5) at a fixed point are quadratic homogenous equations in the couplings and in $g$. The former property allowed us to take the couplings very small and to reduce the moduli space to $\mathscr{C}=R P^{2}$. There are two types of points (phases) in this moduli space, those with and without broken replica symmetry.

Though we have found all IR-fixed points of the one-loop $\beta$ function, the stability of the nontrivial fixed points and of the analytic RG flow to them remain unexplored. Finally, it is interesting to generalize this analysis to more complex supersymmetric theories and to find realistic models whose critical behavior correspond to such theories.

\section{ACKNOWLEDGMENTS}

I would like to thank D. Ivanov, A. Marshakov and A. Mironov for an an atmosphere of wholehearted support and for helpful conversations. I am especially indebted to 
A. Morozov and I. Polyubin for their stimulating suggestions and comments. Also I am grateful to V. Dotsenko, from whom I first learned what a spin glass is and with whom many of the ideas presented here have been discussed.

This work was supported in part by the Russian Fund for Fundamental Research, Grant No. 96-15-96939.

a)e-mail: gukov@landau.ac.ru

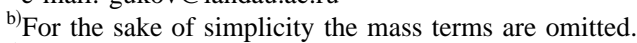

${ }^{c}$ The points where the $\beta$ functions vanish.

d) If $\lambda_{3} \neq 1$ then the correct parameters are $\lambda=\lambda_{2} / \lambda_{3}$ and $\mu=\lambda_{1} / \lambda_{3}$.

e) Otherwise we get a trivial replica-symmetric fixed point.

${ }^{1}$ M. Mezard, G. Parisi and M. A. Virasoro, Spin Glass Theory and Beyond, World Scientific (Singapore 1987).

${ }^{2}$ V. S. Dotsenko, Introduction to the Theory of Spin-Glasses and Neural Networks, World Scientific (Singapore 1994).

${ }^{3}$ Vl. Dotsenko, M. Picco and P. Pujol, "Two critical models with disorder," PAR-LPTHE-95-63, hep-th/ 9512087.

${ }^{4}$ G. Parisi and N. Sourlas, "Random magnetic fields, supersymmetry, and negative dimensions," Phys. Rev. Lett. 43 (1979), 744

${ }^{5}$ J. Kurchan, Supersymmetry in Spin Glass Dynamics, 1993.

${ }^{6}$ N. Seiberg, "The power of holomorphy: exact results in 4D SUSY field theories," PASCOS 1994, 357-369, hep-th/9408013.

${ }^{7}$ K. Intriligator and N. Seiberg, "Lectures on supersymmetric gauge theories and electric-magnetic duality," Nucl. Phys. Proc. Suppl. 45BC: 1-28 (1996), hep-th/9509066.

${ }^{8}$ J. Wess and B. Zumino, “A Lagrangian model invariant under supergauge transformations,” Phys. Lett. 49B (1974), 52.

Published in English in the original Russian journal. Edited by Steve Torstveit. 